\title{
Correction to: Constraints on Janus Cosmological model from recent observations of supernovae type la
}

\author{
G. D'Agostini ${ }^{1}$ (D) J.P. Petit ${ }^{1}$
}

Published online: 25 June 2018

(c) Springer Nature B.V. 2018

\section{Correction to: Astrophys Space Sci (2018) 363:139 https://doi.org/10.1007/s10509-018-3365-3}

The original article has been updated for copyright reasons with Fig. 1 and a too vague formulation intended to give credit to Farnes (2017) for the originally displayed figure. The sentences

"JCM explains the confinement of galaxies and their flat rotation curves, as recently showed by Farnes (2017), see Fig. 1. Mysterious dark matter is no longer required, while the mainstream $\triangle C D M$ model does." have been replaced by

"JCM explains the confinement of galaxies and the shape of their rotation curves. As we showed in Petit et al. (2001), if one introduces a surrounding repellent negative matter environment, it gives larger rotation velocities at distance, see Fig. 1. Mysterious dark matter is no longer required, while the mainstream $\Lambda C D M$ model does."

Figure 1 has been replaced. The figure caption of the earlier Fig. 1 has been replaced by

"Fig. 1 Circular velocity, after Petit et al. (2001). This can be compared with results from numerical simulations by Farnes (2017)"

The original article has been corrected.

The online version of the original article can be found under https://doi.org/10.1007/s10509-018-3365-3.

\footnotetext{
$凶$ G. D’Agostini

DAgostiniGilles@mailaps.org

J.P. Petit

Jp.petit@mailaps.org

1 BP 55, 84122 Pertuis, France
} 